\title{
L'enzyme G6PD des globules rouges humains : une chimère
}

\begin{abstract}
Akira Yoshida (city of Hope, Duarte, CA, USA) consacre la plus grande part de ses recherches à la glucose 6- phosphate déshydrogénase depuis plus de 25 ans. Cette enzyme a été largement étudiée dans le monde depuis bien longtemps car son déficit dans les globules rouges humains est responsable de la plus fréquente des maladies enzymatiques, une anémie hémolytique dont la forme la plus habituelle est celle de poussées d'hémolyse intravasculaire déclenchée par des médicaments (notamment des antipaludéens) ou, dans les pays méditerranéens, par les fèves. Plusieurs centaines de millions d'hommes sont atteints de cette affection transmise sur un mode récessif lié à l'X. Cette haute prévalence du déficit est très probablement liée à une résistance conférée aux femmes hétérozygotes contre le paludisme.
\end{abstract}

La base moléculaire du déficit enzymatique est constituée par des mutations ponctuelles du gène situé sur le bras long du chromosome X (Xq28), mutations nombreuses dont chacune peut aboutir à la synthèse d'une enzyme anormale, instable ou (et) dont l'activité catalytique est profondément altérée. Ce préambule explique que la protéine enzymatique des globules rouges humains ait été purifiée très tôt... par Akira Yoshida et son équipe[1], puis que sa séquence ait été analysée par les techniques de séquençage protéique au cours d'un long travail de plus de 15 ans, ayant abouti à la publication en 1986 de l'enchaînement complet des acides aminés [2, 3].

La même année était publiée la séquence complète de l'ADNc par des équipes italiennes ( $\mathrm{Naples}$ ) et anglaise (Londres) animées par un sur cette enzyme et son déficit, Lucio Luzzato [4]. L'identité de cet ADNc ne fait aucun doute car il correspond effectivement au gène de la G6PD sur le chromosome X et est muté chez les malades souffrant du déficit enzymatique $\left(\mathrm{m} / \mathrm{s}, \mathrm{n}^{\circ} 6\right.$, vol. 5, p. 432).

La comparaison des séquences d'acides aminés déduite de l'enchâ̂nement des nucléotides de l'ADNc [4] ou déterminée directement $[2,3]$ devait cependant déboucher sur une grande surprise. L'ADNc a le potentiel de coder pour une sous-unité de 515 résidus. Les 479 acides aminés carboxyterminaux déterminés par séquençage protéique correspondent bien à la séquence nucléotidique, mais pas les 53 résidus aminoterminaux qui n'ont aucun rapport avec la séquence des 36 premiers résidus déduite de celle de l'ADNc (figure 1). Réanalysant ses données dans un récent article publié dans Cell, A. Yoshida et ses collègues confirmèrent la séquence protéique préalablement publiée, mais démontrèrent aussi que cette espèce de sous-unité de G6PD coexistait avec une espèce moins abondante qui pouvait intégralement correspondre à la séquence déduite de l'ADNc [5] (figure 1). Une sonde oligonucléotidique spécifique de l'extrémité aminoterminale de la séquence érythrocytaire majoritaire permit d'isoler des clones génomiques et d'ADNc. Le gène codant pour cette séquence est localisé sur le chromosome 6 , l'ADNc ayant le potentiel de coder pour une protéine de 345 acides aminés dont les 54 premiers sont ceux formant le peptide aminoterminal de la forme majeure de l'enzyme érythrocytaire mais dont les suivants, du côté carboxyterminal, ne correspondent à rien de connu.
L'ARN messager de cette protéine hypothétique est présent, en faible quantité, de façon variable selon les tissus; il est relativement abondant dans les réticulocytes (précurseurs des globules rouges) et absent des lymphoblastes. Sa taille est de 1,5 kb, contre 2,4 $\mathrm{kb}$ pour l'ARNm du gène lié à l'X. La G6PD érythrocylaire humaine apparaît donc, selon ces résultats, être une protéine hybride dont les 53 ou 54 premiers acides aminés (le résidu 54, une méthionine, peut correspondre au gène du chromosome 6 , mais auss̀i à celui du chromosome X dont le codon 36 est également une méthionine, voir figure 1) sont codés par un gène autosomique et les 478 ou 479 résidus suivants par le gène de la G6PD lié à l'X (figure 1).

Deux mécanismes décrits dans d'autres systèmes peuvent être ici éliminés d'emblée : l'épissage croisé (transsplicing) et «l'édition sur épreuve» (editing) de l'ARN [6]. Le premier processus, décrit notamment chez les trypanosomes et les nématodes, associe des exons issus de gènes différents. Le second mécanisme, caractéristique des mitochondries de trypanosomes et autres kinétoplastes, modifie, parfois considérablement, un transcrit primitif en ajoutant ou en retranchant des résidus uridyliques [7]. Dans ces deux processus, cependant, il y a création d'espèces hybrides ou modifiées d'ARN messager, ce qui est exclu dans les expériences de A. Yoshida et al. : la protection contre la digestion par la nucléase $\mathrm{Sl}$ et des amplifications par PCR (polymerase chain réaction) ne mettent en évidence que les deux espèces d'ARNm décrites dans la figure 1.

Reste trois explications plausibles 
Figure 1. Mécanismes possibles de la formation d'une G6PD chimérique dans les globules rouges humains. Le gène de la G6PD, sur le chromosome $X$, code pour un ARNm ubiquitaire de $2,4 \mathrm{~kb}$, traduit en une sous-unité de 515 acides aminés. Un gène sur le chromosome 6 code pour un ARNm de 1,5 kb qui a le potentiel d'être traduit en une protéine inconnue (et dont l'existence n'a pas été démontrée) de 345 acides aminés. Dans les globules rouges humains, on trouve le produit attendu du gène G6PD (forme mineure) et une espèce hybride, majeure, dans laquelle les 35 premiers acides aminés de l'enzyme entièrement codée par l'ARNm de la G6PD ont été remplacés par les 53 résidus aminoterminaux du produit présomptif de traduction de I'ARNm autosomique. Cette molécule chimérique pourrait être le résultat d'une "traduction croisée », débutant sur le messager autosomique et se poursuivant sur le messager G6PD. Ces deux messagers pourraient s'assembler notamment grâce à la présence dans leurs parties $5^{\prime}$ non codantes (rectangle blanc) d'une petite zone de 15 bases dont 13 sont identiques dans les deux messagers (régions grisées). Un processus post-traductionnel de transpeptidation est également possible. En noir, région codante de I'ARN messager du gène autosomique et protéine présomptive correspondante. En rouge, région codante de I'ARN messager du gène lié à I'X et protéine correspondante.

auxquelles il est possible de penser à ce jour: (1) l'appareil de traduction protéique commencerait à lire l'ARNm autosomique jusqu'à la méthionimie 54 , là il « sauterait » sur la méthionine 36 de l'ARNm de la G6PD et achèverait la traduction. Quoiqu'on ne connaisse pas d'exemple semblable de traduction croisée de deux ARNm, on sait que le ribosome peut en effet, sur un même messager, «sauter » une base, modifiant ainsi une phase de lecture, voire court-circuiter plusieurs codons [6] ; (2) la fusion pourrait se produire entre deux polypeptides préalablement synthétisés, selon un mécanisme, dont il n'existe pas non plus d'exemple, de «transpeptidation ». Un argument en faveur d'un tel phénomène, bien indirect, est que l'enzyme dont la séquence a été analysée par l'équipe de Yoshida provient des globules rouges, cellules dépourvues $\mathrm{m} / \mathrm{s} n^{\circ} 8$ vol. 5 , octobre 89
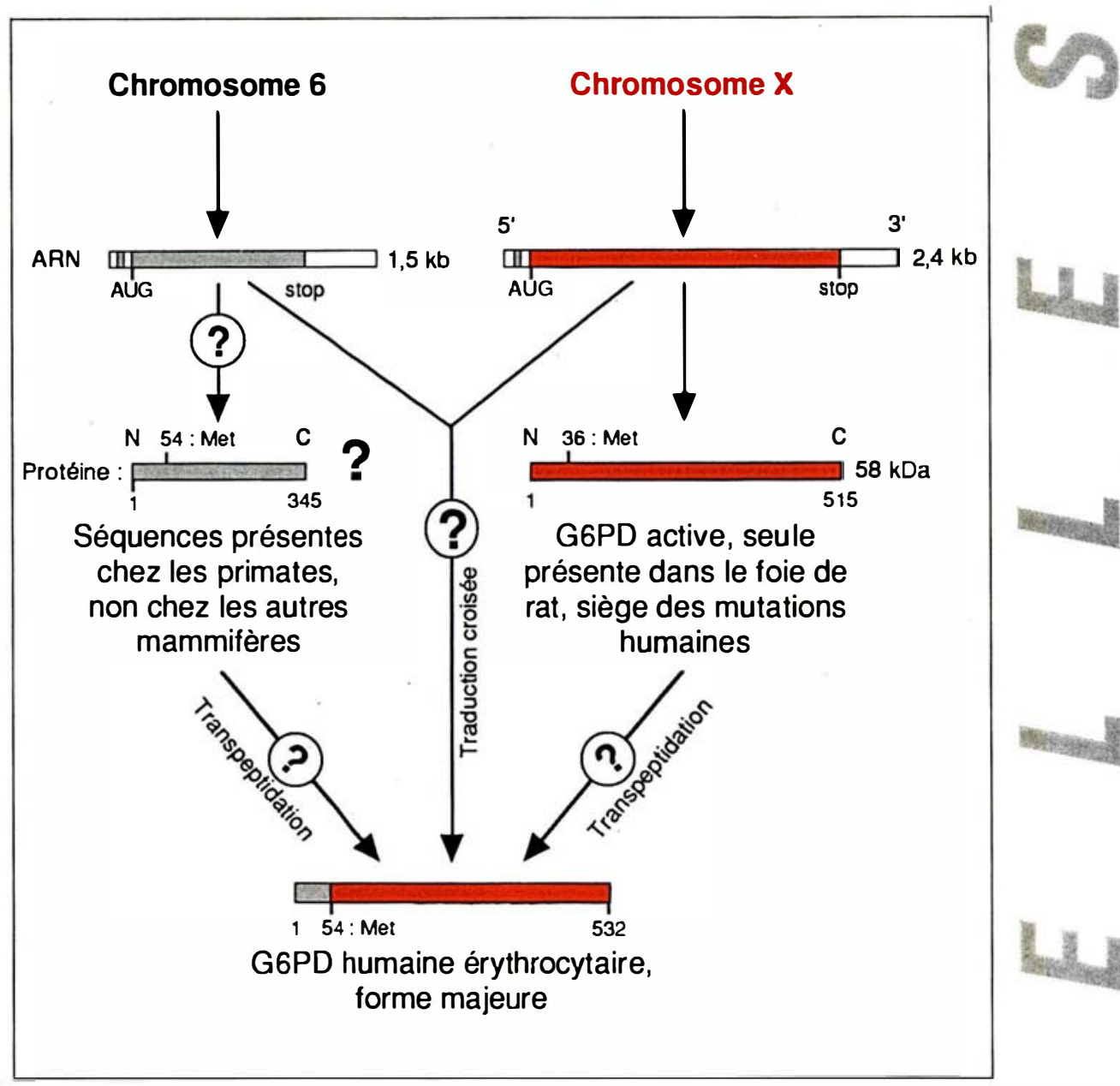

de synthèse protéique et vivant jusqu'à 120 jours, possédant donc dès leur naissance un stock d'enzymes qui pourront subir, au fil des mois, des modifications post-traductionnelles relativement importantes [8]. Pour tester ccette hypothèse, il faudrait étudier l'extrémité aminoterminale de la G6PD de cellules à renouvellement rapide et à synthèse protéique active, par exemple les leucocytes [8], ou comparer les proportions entre la forme majeure et la forme mineure de G6PD dans des globules rouges jeunes ou vieux ; (3) on ne peut, enfin, éliminer totalement à ce jour l'existence d'un artéfact encore indéfini. Plusieurs observations sont en effet extrêmement troublantes. La G6PD de foie de rat a la structure attendue pour un produit entièrement codé par le gène lié à l'X... et est pleinement active. Peutêtre cela est-il dû à la synthèse per- manente dans ce tissu d'une enzyme à renouvellement rapide, et qui n'a donc pas le temps de subir une éventuelle transpeptidation post-traductionnelle. Une autre possibilité, proposée par Yoshida, est que le gène situé sur le chromosome 6 n'existerait pas chez le rat. De fait, les sondes spécifiques de cette séquence détectent des séquences homologues chez les primates... mais pas chez les autres mammifères.

Quoiqu'il en soit, la signification éventuelle d'une telle création de molécules hybrides par la mise en place d'un processus complexe récemment acquis dans l'évolution reste bien obscure, d'autant que le gène humain localisé sur le chromosome X suffit à lui tout seul pour gouverner la synthèse d'une G6PD parfaitement active dans des cellules humaines [6]. Quelle que soit par conséquent le prestige de l'équipe .

(2)

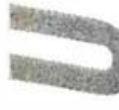


d'Akira Yoshida (le poids de son article étant encore renforcé par une autre signature célèbre, celle de Y.W. Kan), il faut probablement attendre les résultats de quelques expériences complémentaires pour considérer comme acquises les données discutées ici. Les ADNc correspondant aux gènes autosomiques et liés à l'X pourraient ainsi être transcrits in vitro en ARN qui pourraient être traduits ensemble dans un système de traduction acellulaire... par exemple de réticulocyte. Il serait alors possible de déterminer si un processus de «traduction croisée » peut être observé. L'utilisation d'anticorps spécifiques de l'extrémité aminoterminale de la forme majeure de G6PD érythrocytaire permettrait aussi, en utilisant des techniques d'immunotransfert (Western blot), de vérifier que la protéine hybride existe bien in vivo et n'est pas la conséquence d'une altération survenant in vitro.

A.K.

1. Yoshida A. Glucose-6-phosphate dehydrogenase of human erythrocyte. Purification and characterization of normal $(\mathrm{B}+)$ enzyme. J Biol Chem 1966; 241 : 4966-76.

2. Takizawa $T$, Huang IY, Ikuta $T$, Yoshida A. Human glucose-6-phosphate dehydrogenase: primary structure and DNA cloning. Proc Natl Acad Sci USA $1986 ; 83$ : 4157 . 61.

3. Yoshida A, Huang IY. Structure of human glucose-6- phosphate dehydrogenase. In Yoshida A, Beutler E, eds. Glucose 6- phosphate dehydrogenase. Orlando: Academic Press, 1986 ; 473-82.

4. Persico MG, Vigietto G, Martini G, et al. Isolation of human glucose -6- phosphate dehydrogenase (G6PD) cDNA clones: primary structure of the protein and unusual 5 ' noncoding region. Nucleic Acids Resl, $1986 ; 14: 2511-22$ (correction dans $14: 7822$ ). 5. Kanno H, Huang IY, Kan YW, Yoshida A. Two structural genes on different chromosomes are required for encoding the major subunit of human red cell glucose -6- phosphate dehydrogenase Cell 1989; 58 : 595-606. 6. Borst P, Benne R, Tabak HF. A fused chimeric protein made in human cells. Cell $1989 ; 58: 421-2$.

7. Simpson L, Shaw J. RNA editing and the mitochondrial cryptogenes of kinetoplastid protozoa. Cell 1989; 57 : 355-66.

8. Kahn A, Bertrand O, Cottreau D, Boivin $P$, Dreyfus JC. Evidence for structural differences between human glucose-6- phosphate dehydrogenase purified from leukocytes and erythrocytes. Biochim Biophys Res Commun $1977 ; 77: 65-72$

\section{BRËVES 口D}

Génétique des anomalies de la vision des couleurs : monochromasie bleue par lésion d'une séquence d'ADN à distance des gènes codant pour les pigments rouge et vert. Les pigments responsables de la vision des couleurs rouge et verte sont codés par des gènes aux séquences très voisines localisés sur le chromosome $\mathrm{X}$ alors que le pigment «bleu » est codé par un gène autosomique. Les gènes localisés sur le chromosome $\mathrm{X}$ sont modifiés, le plus souvent par recombinaison homologue inégale, chez les dyschromates du type des daltoniens $\left(m / s\right.$ suppl. au $n^{\circ} 7$, vol. 3, p.20). Deux types de monochromates «bleu», c'est-à-dire de malades ne voyant que le bleu et n'ayant aucune sensibilité aux couleurs rouge et verte, ont été distingués par J. Nathans et ses collègues (Baltimore, MD, LISA) [1, 2]. Les uns sont des malades ayant deux lésions : d'une part un remaniement des gènes «rouge » et « vert » de type dischromasie, et une mutation ponctuelle surajoutée du gène actif restant. Les autres n'ont pas de modification des gènes «rouge » et «vert », mais une délétion située en amont d'eux, intéressant notamment une séquence de 579 paires de bases (pb) localisée à 4 kilo bp (kpb) du gène « rouge » et $43 \mathrm{kpb}$ du gène "vert» le plus proche. Il est probable que cette séquence correspond ̀̀ un élément régulateur indispensable à l'expression normale de tous les gènes du locus, rappelant en cela les $\beta$-thalassémies dans lesquelles la délétion de l'élément activateur situé à 45 kpb en amont du gène $\beta$ de la globine empêche l'expression de tous les gènes du locus $\beta$ qui ne sont pas intéressés par cette délétion $(\mathrm{m} / \mathrm{s}$, $n^{\circ} 4$, vol. 4, p. 252).

[1. Nathans J, et al. Science 1989 ; 245 : 831-8.].

Le système du complément pourrait être activé par les oligodendrocytes, les cellules gliales qui, dans le système nerveux central, protègent les axones et les myélinisent [1].
Lorsque des cellules provenant d'un nerf optique de rat sont mis en présence, in vitro, de sérum de rat, des complexes lytiques transmembranaires sont rapidement observés sur les oligodendrocytes, provoquant la destruction des cellules. Cette activation de la voie alterne du complément - ne nécessitant pas la présence d'anticorps - a été étudiée plus précisément par l'incubation des oligodendrocytes avec des doses sublétales de sérum de rat [2]. Grâce à cette technique, l'attaque du complément est observée, sous la forme de nombreuses vésicules accolées à la membrane des oligodendrocytes, mais l'absence de lyse rapide permet une identification des éléments en cause. L'analyse immunocytochimique des vésicules a permis d'identifier la molécule oligodendrocytaire impliquée dans le complexe lytique ; il s'agit d'un polysaccharide, le galactocérébroside, qui est un composant habituel de la membrane de ces cellules. Cette activation du système du complément par les cellules myélinisantes du système nerveux central pourrait être une clé pour comprendre la sclérose en plaque (SEP). Dans les mêmes travaux, des éléments du liquide céphalo-rachidien ont pu être marqués par des anticorps anti-fractions terminales du complément et anti-galactocérébroside chez des malades atteints de SEP, suggérant la présence des vésicules. Les conditions dans lesquelles se déroule cette activation restent bien sûr à définir. Un point semble établi, cependant, c'est qu'une telle activation requiert la rupture de la barrière hémato-encéphalique qui, normalement, prévient totalement l'entrée des facteurs du complément dans le parenchyme cérébral et donc le contact avec les oligodendrocytes. La SEP serait-elle primitivement une maladie de la barrière?

[1. Scolding NJ, et al. J Neurol Sci $1989 ; 89$ : 289-300.]

[2. Scolding NJ, et al. Nature 1989 ; 339 : 620-2.] 